\title{
Evaluación del efecto de la precarga de ensamble y la cantidad de hojas sobre la disipación pasiva de energía en un muelle
}

\author{
Evaluation of the effect of assembly preloads and the number of leaves on \\ passive energy dissipation of a leaf spring
}

\author{
Víctor Iván Rodríguez Reyes ${ }^{\circledR}$, Dariusz Slawomir Szwedowicz
}

Tecnológico Nacional de México / Centro Nacional de Investigación y Desarrollo Tecnológico (CENIDET), Interior Internado Palmira, CP. 62490, Cuernavaca, Morelos, México

\begin{abstract}
Autor de correspondencia: Víctor Iván Rodríguez Reyes, Tecnológico Nacional de México / Centro Nacional de Investigación y Desarrollo Tecnológico (CENIDET), Interior Internado Palmira, CP. 62490, Cuernavaca, Morelos, México. Email: victor.rodriguez18me@ cenidet.edu.mx. ORCID: 0000-0002-4984-025X.
\end{abstract}

Recibido: 01 de Octubre del 2020 Aceptado: 07 de Noviembre del 2020 Publicado: 09 de Noviembre del 2020

Resumen. - En este trabajo se presenta la evaluación de la influencia de las precargas de ensamble y de la cantidad de hojas en el fenómeno de disipación de energía de un muelle a través de un estudio paramétrico. El estudio consistió en estimar la cantidad de energía disipada al variar el número de hojas del muelle y la precarga, a través del uso de abrazaderas, tanto de forma numérica como experimental. Mediante ensayos de compresión se observó el comportamiento del muelle mediante una relación fuerza-desplazamiento. Se obtuvo la energía de deformación y la disipación por fricción total mediante la integración de la curva de histéresis. Se realizó un modelado numérico con el paquete comercial de elementos finitos Abaqus en estado cuasi estático. Los resultados obtenidos demuestran que las precargas influyen en la disipación de energía del muelle, incrementándose mayormente cuando éstas se implementan con un mayor número de hojas, hasta 189\%. Con estos resultados es posible proponer una configuración que permite disipar la mayor cantidad de energía de forma pasiva y, en consecuencia, mejorar el amortiguamiento del vehículo en referencia al muelle comercial.

Palabras clave: muelle de hojas; efectos de precargas; disipación pasiva de energía; elementos finitos

\begin{abstract}
This work presents the evaluation of the influence of the assembly preloads and the number of leaves on the energy dissipation phenomenon of a leaf spring through a parametric study. The study consisted of estimating the amount of energy dissipated by varying the number of spring leaves and preloads, through the use of clamps, both numerically and experimentally. Through compression tests, the behavior of the spring was observed through a force-displacement relationship. The strain energy and total frictional dissipation were obtained by integrating the hysteresis curve. Numerical modeling was performed with the commercial finite element package Abaqus in a quasi-static state. The results obtained show that the preloads influence the energy dissipation of the spring, increasing mainly when these are implemented with a greater number of leaves, up to $189 \%$. With these results, it is possible to propose a configuration that allows the greatest amount of energy to be dissipated passively and, consequently, to improve the damping of the vehicle in reference to the commercial spring.
\end{abstract}

Keywords: leaf spring; effect of preloads; passive dissipation of energy, finite element analysis 


\section{Revista de Ciencias Tecnológicas (RECIT). Volumen 3 (4): 181-195}

\section{Introducción}

Existen diferentes métodos para inhibir los efectos de las cargas de impacto y vibraciones. Estos se clasifican en tres grupos: eliminación de la fuente de vibración, aislamiento del sistema, y reducción de la respuesta [1]. Entre éstos, la reducción de la respuesta del sistema por medio de la disipación de energía se caracteriza por su efectividad, economía, y rapidez de implementación. Estos medios de disipación pueden ser activos, semiactivos o pasivos. La característica de los sistemas activos es que requieren de energía de entrada para su funcionamiento porque requieren de actuadores, servomotores y sistemas de retroalimentación. En cambio, los sistemas pasivos no requieren de energía de entrada, ya que el amortiguamiento se da gracias a las propiedades intrínsecas de los materiales y del diseño del sistema mecánico. El denominado control semiactivo es una combinación de ambos, el cual requiere un consumo menor de energía.

Para el control de los efectos de impacto o vibraciones por disipación pasiva de energía, se utilizan medios friccionantes, viscosos, viscoelásticos, y de deformación plástica. La disipación por fricción seca se caracteriza por su efectividad, disponibilidad y bajo costo. En estos sistemas, la disipación de energía se da debido al movimiento relativo entre dos superficies. Algunos de los sistemas más comunes de disipación por fricción seca son el sistema de arriostramiento Pall, la tijera Truffault y el muelle de fricción [2].

El muelle de hojas, también conocido como ballesta, consiste en una serie de hojas de acero de diferentes longitudes, las cuales están apiladas unas sobre otras. Una de ellas, la hoja maestra, tiene en los extremos ojales mediante los cuales el muelle se monta al chasis de un vehículo. Los muelles presentan buenas características de costos, durabilidad, y fácil mantenimiento [3], pero, por otra parte, entre sus desventajas se encuentran características dinámicas bajas y ruido [4]. Los muelles absorben energía, la almacenan y posteriormente la liberan, además de que presentan una disipación de energía derivada del deslizamiento relativo de las hojas en contacto. Los muelles siguen siendo un medio efectivo para la disipación de energía ante impactos o vibraciones, aunque su campo de aplicación se ha visto reducido en su mayoría a vehículos pesados como furgonetas, camiones, vehículos todoterreno y remolques.

Se ha buscado mejorar las características de los muelles, variando la rigidez con diseños que modifican su geometría [5]-[8]. También se han realizado trabajos de diseño y manufactura de muelles empleando materiales compuestos, sustituyendo a los aceros convencionales para muelle, lo que mejora su desempeño, aunque con inconvenientes constructivos [9]-[12]. Por otra parte, cuando se diseña un muelle se suelen realizar algunas simplificaciones, e incluso en aquellos donde se emplea el método de elementos finitos, los modelos han presentado deficiencias [2]. Una de ellas es que no se considera la fricción por el contacto entre las hojas, modelándose con la teoría de vigas de la mecánica de sólidos como media viga en voladizo. Por lo tanto, se ha buscado el desarrollo de modelos que consideren la influencia del contacto entre las hojas. Qin et al. [13] modelaron muelles en una suspensión Hotchkiss bajo diversos escenarios de carga, determinando diversas características de la suspensión. Emplearon

ISSN: 2594-1925 
diferentes tipos de elementos finitos de un paquete comercial que permitiera incluir contacto entre las hojas, consiguiendo una buena correlación con resultados de pruebas de laboratorio, proponiendo sus metodologías para el desarrollo de muelles y suspensiones previo a la etapa de prototipos. Kadziela et al. [14] modelaron dos configuraciones diferentes de montaje de muelles. La primera reprodujo el montaje real del muelle en un vehículo, mientras que en la segunda se simplificaron varios elementos del ensamble. Esto permitió una mejor evaluación del muelle sin la influencia de las sujeciones. Demostraron que su enfoque puede ser usado para modelar apropiadamente el comportamiento de muelles, alcanzando mejor convergencia la configuración simplificada. Krason et al. [15] modelaron un muelle, determinando que hay una influencia de las condiciones superficiales de las hojas en la aceleración vertical del vehículo, mientras que la transmisibilidad de las vibraciones no depende solamente de la condición superficial, si no de los parámetros de excitación. Krason y Wysocki [16] realizaron la medición de temperatura de una suspensión de automóvil con una cámara térmica, con la cual demostraron que es posible localizar detalladamente las zonas donde se presenta mayor fricción, indicando disipación de energía. Krason et al. [17] evaluaron el efecto de la fricción debido al contacto entre hojas de un muelle de doble etapa, y el papel que los claros y la fricción juegan en la disipación de energía. Con una cámara térmica infrarroja observaron las zonas donde se presentaba menor y mayor calentamiento, relacionándolas con la disipación de energía, observando su relación con las áreas de contacto en un estudio numérico.
En este trabajo se analizó la influencia de la cantidad de hojas y de la implementación de precargas en la disipación de energía del muelle por fricción seca, a través de pruebas cuasiestáticas de laboratorio y el método de elementos finitos, con el propósito de proponer una configuración que permita mejorar el amortiguamiento por disipación pasiva de energía, cuando se compara con el modelo comercial usado en la suspensión de un remolque.

\section{Metodología}

Se realizó una evaluación numérica con el paquete de elementos finitos Abaqus, con el fin de valorar su factibilidad para modelar el fenómeno de disipación de energía de un muelle. Se efectuó un estudio experimental cuasiestático en una máquina universal, que permitió validar los resultados arrojados los modelos discretos.

\subsection{Materiales y métodos}

El muelle comercial empleado en este estudio es de la marca MAF modelo QTR224, semielíptico y simétrico, de cuatro hojas: una hoja maestra y tres graduadas. Este es un diseño empleado para remolques de $1088 \mathrm{~kg}(2400 \mathrm{lb})$ y está fabricado de acero para muelle AISI 5160. La longitud total, de ojal a ojal, es de $668 \mathrm{~mm}(26$ $5 / 16$ "). Las propiedades elásticas de dicho acero se presentan en la Tabla 1. Este diseño se monta en el eje del remolque y sirve tanto como elemento de amortiguamiento como elemento estructural.

Tabla 1. Propiedades del acero AISI 5160.

\begin{tabular}{cc} 
Módulo de elasticidad (GPa) & 205 \\
Relación de Poisson & 0.29 \\
${\text { Densidad }\left(\mathbf{k g} / \mathbf{m}^{3} \text { ) }\right.}^{\text {Denside }}$ & 7850 \\
Esfuerzo de fluencia (MPa) & 1005 \\
Esfuerzo último (MPa) & 1145 \\
\hline
\end{tabular}


Para implementar una precarga que ejerza un apriete entre las hojas, se confeccionaron sujeciones con elementos hechos de solera de acero y pernos. Los elementos de apriete se fabricaron con solera de acero de 25.4 $\mathrm{mm}$ de ancho (1") por $3.175 \mathrm{~mm}$ de espesor $\left(1 / 8^{\prime \prime}\right)$. Éstos se recortaron a una longitud de
$85 \mathrm{~mm}$, distancia suficiente para que estos elementos entren en contacto con las hojas del muelle y sean apretados por dos tornillos M10 grado 5. Este tipo de tornillos tiene un torque máximo de apriete de $55 \mathrm{~N}-\mathrm{m}$. Estos elementos se colocaron a lo largo del muelle, como se observa en la Figura 1.

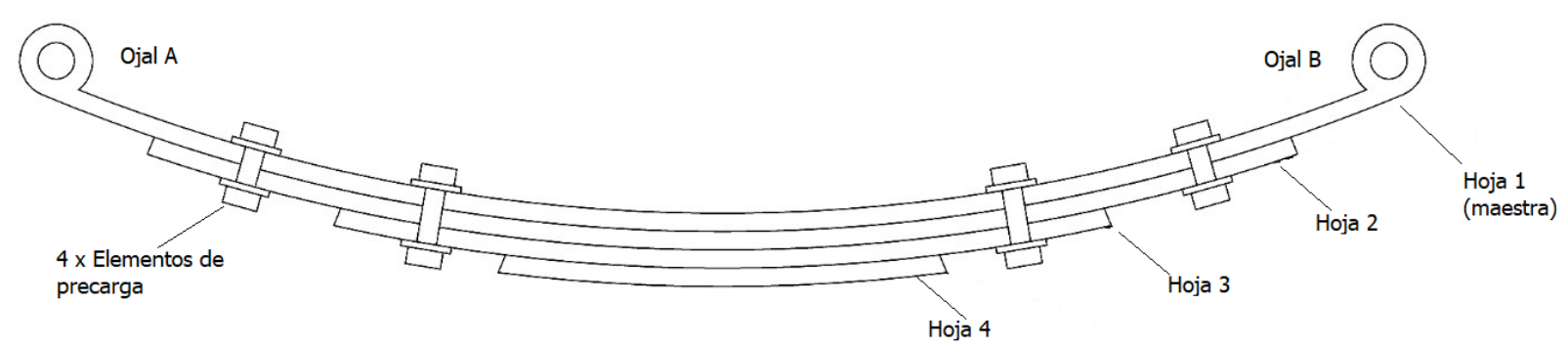

Figura 1. Configuración para la evaluación del muelle.

Se realizó un estudio paramétrico para evaluar experimentalmente el efecto de la cantidad de hojas y el apriete de precarga, representado por la cantidad de abrazaderas, por separado. Para cada uno de estos factores se trabajó con dos niveles, un mínimo de dos hojas y un máximo de cuatro, y un mínimo de dos abrazaderas y un máximo de cuatro. Los detalles de las configuraciones se muestran en las cuatro primeras filas de la Tabla 2.

Para efectos de extender el estudio a otras configuraciones, las últimas tres filas de la Tabla 2 representan pruebas realizadas sólo numéricamente. Con esto se obtuvieron puntos extras para el trazado de una gráfica tridimensional que relacione la disipación de energía con las variables del experimento.
Tabla 2. Diseño del estudio paramétrico. La abreviación Exp. indica evaluación experimental y Num. evaluación numérica.

\begin{tabular}{ccccc}
\hline Configuración & Exp. & Num. & $\begin{array}{c}\text { Cantidad } \\
\text { de hojas }\end{array}$ & $\begin{array}{c}\text { Cantidad } \\
\text { de precargas }\end{array}$ \\
\hline 2H2P & $\times$ & $\times$ & 2 & 2 \\
2H4P & $\times$ & $\times$ & 2 & 4 \\
4H2P & $\times$ & $\times$ & 4 & 2 \\
4H4P & $\times$ & $\times$ & 4 & 4 \\
3H0P & & $\times$ & 3 & 0 \\
3H2P & & $\times$ & 3 & 2 \\
3H4P & & $\times$ & 3 & 4 \\
\hline
\end{tabular}

\subsection{Estudio experimental}

Se evaluó la influencia en la disipación de energía por fricción seca del muelle de los cambios en parámetros como el número de hojas que lo conforman, y la implementación de precargas, que se representan físicamente mediante elementos de apriete con pernos.

La disipación de energía se estima al calcular el área entre la curva fuerzadesplazamiento de un ciclo completo de carga y descarga. Al modificar las condiciones de presión con elementos con contacto superficial, la energía disipada cambia en cada configuración, 
principalmente por el cambio en las condiciones de fricción y rigidez del sistema. Por otro lado, al modificar la cantidad de hojas se incrementa la rigidez del muelle y, por lo tanto, la pendiente de la curva fuerza-desplazamiento.

El muelle se montó en un banco con un canal $\mathrm{C}$ de acero. Un extremo es fijo, mientras que en el otro se permite un movimiento longitudinal libre. Se aplicaron los elementos de precarga en el muelle y se

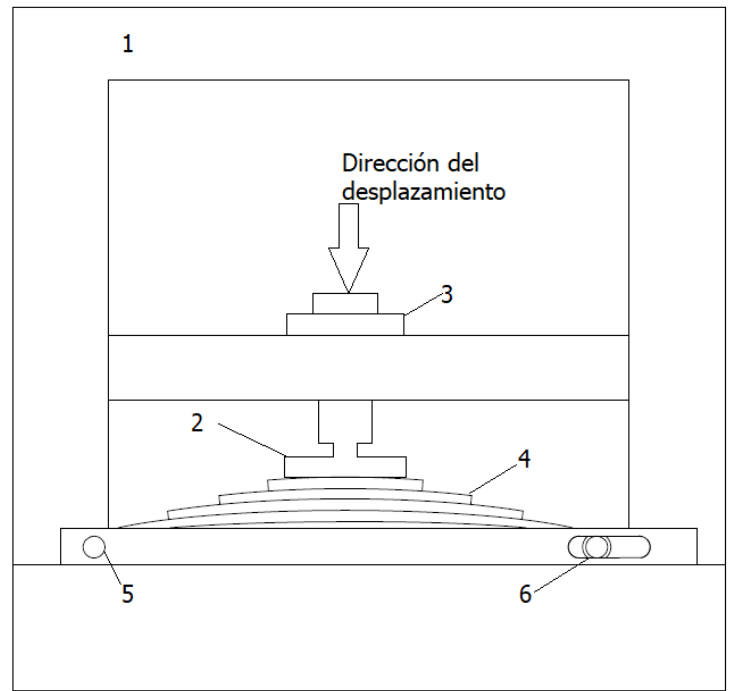

Figura 2. Desarrollo experimental, donde: 1 Shimadzu Autograph AG-X plus 100 kN, 2 - plato de compresión, 3 - celda de carga, 4 - muelle de hojas, 5 - ojal A fijo, 6 - ojal B móvil. varió el número de estos. La evaluación experimental se realizó de acuerdo con la guía del Manual de Diseño SAE HS788 [18]; los ensayos se llevaron a cabo en una máquina universal Shimadzu Autograph AG-X plus $100 \mathrm{kN}$ (ver Figura 2), aplicando un desplazamiento vertical de $35 \mathrm{~mm}$ en el punto medio del muelle, con el cual se alcanza una carga similar a la recomendada por el fabricante, a una velocidad de 5 $\mathrm{mm} / \mathrm{min}$, para posteriormente volver a cero.

La curva de histéresis obtenida de los diagramas fuerza-desplazamiento de las pruebas se integró numéricamente de acuerdo con la ecuación (1), dado que el área entre la curva es la energía disipada $E_{r}$ por la fricción seca, siendo $P$ la carga aplicada, $f_{\max }$ el valor máximo de la deflexión del muelle, $f_{0}$ el valor inicial y $f_{k}$ el valor final [17]. La energía potencial o elástica $E_{o}$, debida a la deflexión del muelle al someterlo a la carga, se obtuvo por la integración numérica de la ecuación (2), donde igualmente $P$ representa a la carga aplicada y $f_{\max }$ el valor máximo de la deflexión alcanzado por el muelle.

$$
\begin{gathered}
E_{r}=\oint_{f} P d l=\int_{f_{0}}^{f_{\max }} P d l+\int_{f_{\max }}^{f_{k}} P d l=\sum_{i=1}^{n-1} \frac{1}{2}\left(P_{i+1}+P_{i}\right)\left(f_{i+1}-f_{i}\right)+\sum_{i=n}^{j-1} \frac{1}{2}\left(P_{i+1}+P_{i}\right)\left(f_{i+1}-f_{i}\right) \\
E_{o}=\oint_{0}^{f_{\max }} P d l=\sum_{i=1}^{n-1} \frac{1}{2}\left(P_{i+1}+P_{i}\right)\left(f_{i+1}-f_{i}\right)
\end{gathered}
$$

La capacidad de amortiguamiento específico, representada por $\psi$, es la cualidad de un material para absorber vibraciones debido a la fricción, 
convirtiendo la energía mecánica en calor. Esta se define de acuerdo con la ecuación (3) como la razón entre la disipación de energía entre la máxima energía potencial durante un periodo de histéresis completo. Este es un parámetro adimensional.

$$
\psi=\frac{E_{r}}{E_{o}}
$$

\subsection{Estudio numérico}

Se realizó un modelo discreto en estado cuasiestático en el paquete comercial de elementos finitos Abaqus, donde se evaluó la energía presentada por la deformación ante un desplazamiento vertical $\delta$ de $35 \mathrm{~mm}$, aplicado en el punto medio del muelle. Se modelaron de manera individual cada una de las piezas del muelle y se les asignaron las propiedades del material, siendo ensambladas posteriormente. El módulo donde se configuran los procedimientos que definen el tipo de análisis a realizar se conoce como Step, donde se seleccionó un procedimiento estático general, creándose uno para la aplicación de las precargas: uno para la compresión del muelle, y otro para el retiro de la carga. Se configuraron interacciones de contacto entre las hojas, mediante un contacto de tipo duro, con una formulación de fricción tipo penalty, con un coeficiente de fricción de 0.46 [4]. La carga que ejerce el perno central se calculó en términos del diámetro del perno $d$ y un factor de par de torsión $K$. De acuerdo con la literatura [19], se puede emplear un factor de $K$ de 0.20 cuando la condición del perno no se puntualiza. Por lo tanto, para el caso del perno central, se aplicaron cargas puntuales tanto en el punto medio superior como en el inferior del muelle de $23.097 \mathrm{kN}$, correspondientes al perno de $9.525 \mathrm{~mm}$ $\left(3 / 8^{\prime \prime}\right)$ de diámetro de grado 5, fuerza equivalente para un torque de $44 \mathrm{~N}-\mathrm{m}$. Para la implementación de precargas se modeló un elemento de apriete de manera simplificada en la forma de un solo sólido, tal como se observa en la Figura 3, en la que las formas irregulares, tales como las cabezas hexagonales de los pernos, de las tuercas, y de la cuerda del vástago se reemplazan por cilindros.

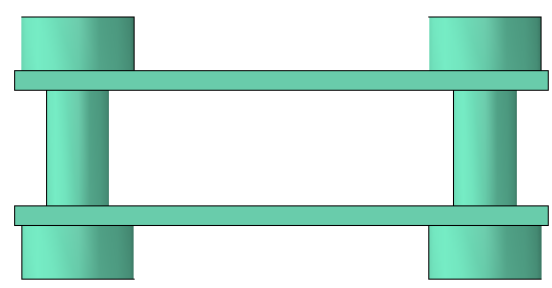

Figura 3. Modelado de elemento para precarga.

Para los tornillos M10 grado 5, se obtuvo una carga interna en el perno de $27.5 \mathrm{kN}$, fuerza equivalente para un torque de $55 \mathrm{~N}$ $\mathrm{m}$ [20]. Para las precargas se aplicaron fuerzas de presión sobre las áreas de las cabezas de los pernos y las tuercas, con magnitud de $110 \mathrm{MPa}$. Las condiciones de frontera se configuran en los ojales. El ojal A, que corresponde al frontal fijo, presenta una condición del tipo perno, por lo que se configuró una condición de rotación alrededor del eje $z$. El ojal B, que corresponde al trasero y es móvil, se configuró con una condición de desplazamiento que permita la traslación a lo largo del eje $x$. La carga para comprimir el muelle se aplicó en el punto central de la hoja más corta. El modelo en 3D empleado para el estudio numérico, con las condiciones de frontera y carga empleadas para simular el muelle se muestra en la Figura 4. El mallado aplicado a las piezas corresponde a un elemento C3D8R, que es un bloque hexaedro de 8 nodos, de la librería standard y de orden lineal. Todos los elementos tienen un tamaño global de

ISSN: 2594-1925 
$6.35 \mathrm{~mm}$, tamaño que se obtuvo tras un análisis de convergencia de malla.

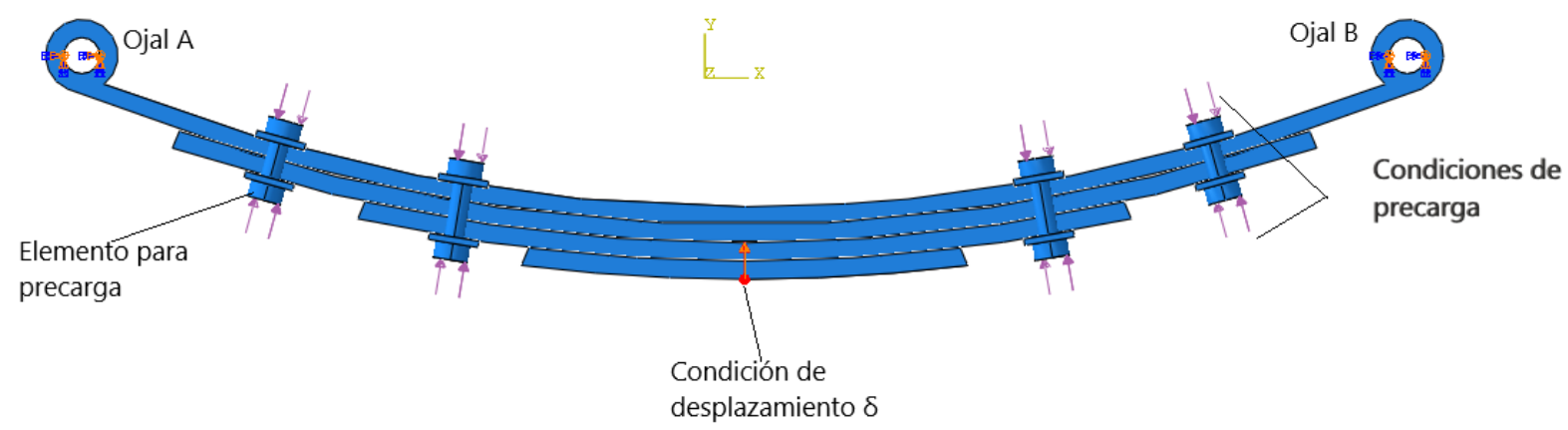

Figura 4. Modelado de elementos de apriete para precarga en hojas del muelle.

Con estas condiciones se observó una variación en la fricción en función de la variación de las cargas normales entre las hojas. Se evaluaron los modelos variando el número de hojas del muelle, de dos a cuatro, y el número de precargas implementadas, de dos a cuatro igualmente. De esta forma, se compararon los resultados de energía de deformación y de disipación por fricción, al igual que se compararon sus respectivas gráficas fuerza-desplazamiento. $\mathrm{Se}$ modelaron los elementos de precarga en diferentes posiciones a lo largo del muelle, de forma que pudieran apretar dos y tres hojas. Estos elementos se colocaron en el modelo en las mismas posiciones que en las pruebas experimentales como se observa en la Figura 4.

\section{Resultados y Discusión}

\subsection{Resultados experimentales}

En la Figura 5 se presentan las curvas de fuerza-desplazamiento obtenidas para las pruebas experimentales con diferentes números de hojas y de abrazaderas, como se describió en la Tabla 2. Se observa el cambio del área entre las curvas, la cual representa la disipación de energía. Se aprecia que, a mayor número de abrazaderas, se requiere de una mayor fuerza para deformar el muelle, lo que indica que éstas también incrementan la rigidez.

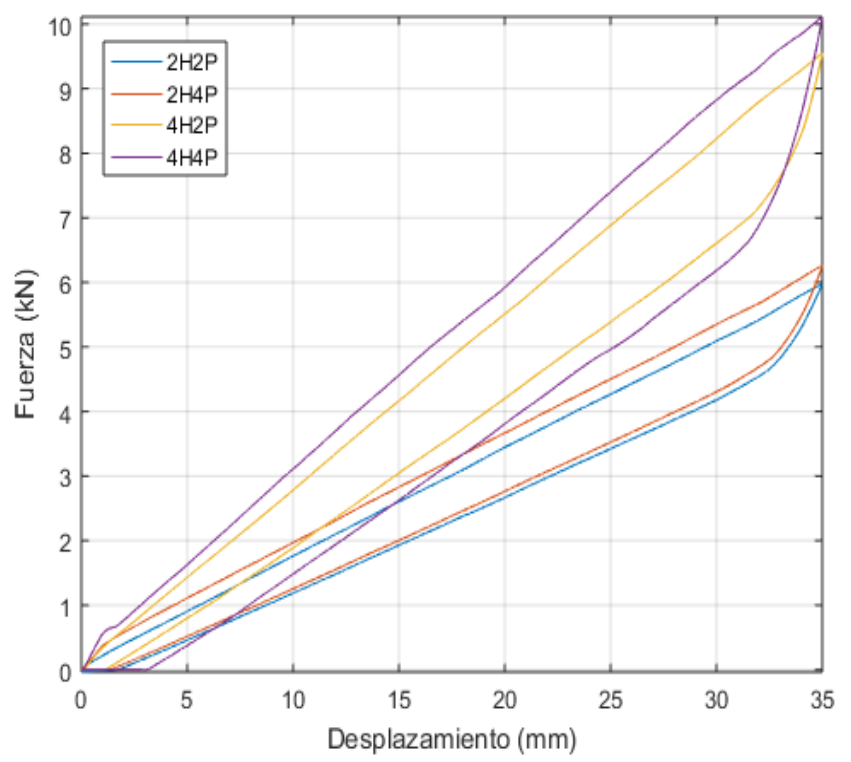

Figura 5. Resultados experimentales de histéresis para muelle.

ISSN: 2594-1925 
Revista de Ciencias Tecnológicas (RECIT). Volumen 3 (4): 181-195

Los datos estimados de la energía elástica $E_{o}$, disipación de energía $E_{r}$, así como de la capacidad de amortiguamiento $\psi$ se presentan en la Tabla 3 . Se observa que existe un aumento significativo en la disipación de energía en función del número de hojas y precargas, mientras que la energía elástica aumenta ligeramente. La disipación de energía $E_{r}$ crece hasta $189 \%$ entre los casos $2 \mathrm{H} 2 \mathrm{P}$ al $4 \mathrm{H} 4 \mathrm{P}$, lo que muestra una tendencia a incrementarse cuando se implementan más precargas en una mayor cantidad de hojas. En el caso de dos hojas, entre las configuraciones $2 \mathrm{H} 2 \mathrm{P}$ y $2 \mathrm{H} 4 \mathrm{P}$ existe un incremento de la disipación de energía de $17 \%$. Para el caso en que se incrementa el número de hojas de dos a cuatro, es decir, de la configuración $2 \mathrm{H} 4 \mathrm{P}$ a 4H2P, el incremento es de 57\%. Finalmente, cuando se trata de cuatro hojas, de la configuración $4 \mathrm{H} 2 \mathrm{P}$ a la $4 \mathrm{H} 4 \mathrm{P}$, hay un incremento de $48 \%$. Se distingue que la tendencia a incrementar de la disipación de energía se da con el aumento del número de hojas. La energía elástica $E_{o}$ presenta incrementos menores, para el caso de dos hojas es de $1.1 \%$ de la configuración $2 \mathrm{H} 2 \mathrm{P}$ a la 2H4P. Al hacer el cambio de dos a cuatro hojas, es decir, de la configuración 2H4P a la 4H2P, la energía elástica se incrementa 55\%. Sin embargo, una vez conformado el muelle por cuatro hojas, entre la configuración 4H2P a la 4H4P hay un incremento de 9\%. Debido al gran incremento de la disipación de energía con respecto a la energía elástica, la capacidad de amortiguamiento específico $\psi$ también presenta un incremento importante.
Tabla 3. Resultados experimentales de la estimación de energía.

\begin{tabular}{cccccc}
\hline Configuración & $\boldsymbol{E}_{\boldsymbol{o}}(\mathrm{J})$ & $\boldsymbol{E}_{\boldsymbol{r}}(\mathrm{J})$ & $\boldsymbol{\psi}$ & $\begin{array}{c}\Delta \boldsymbol{E}_{\boldsymbol{o}} \\
\boldsymbol{\%}\end{array}$ & $\begin{array}{c}\Delta \boldsymbol{E}_{\boldsymbol{r}} \\
\boldsymbol{\%}\end{array}$ \\
\hline 2H2P & 108.085 & 22.162 & 0.21 & - & - \\
2H4P & 109.224 & 25.873 & 0.24 & 1.1 & 17 \\
4H2P & 169.064 & 40.548 & 0.24 & 55 & 57 \\
4H4P & 184.255 & 64.053 & 0.35 & 9 & 48 \\
\hline
\end{tabular}

\subsection{Resultados numéricos}

Empleando el software Abaqus se obtuvieron las curvas de histéresis y se muestran en la Figura 6. Igualmente se observa un incremento del área entre la curva conforme se aumenta el número de precargas, y aún más cuando se aumenta el número de hojas.

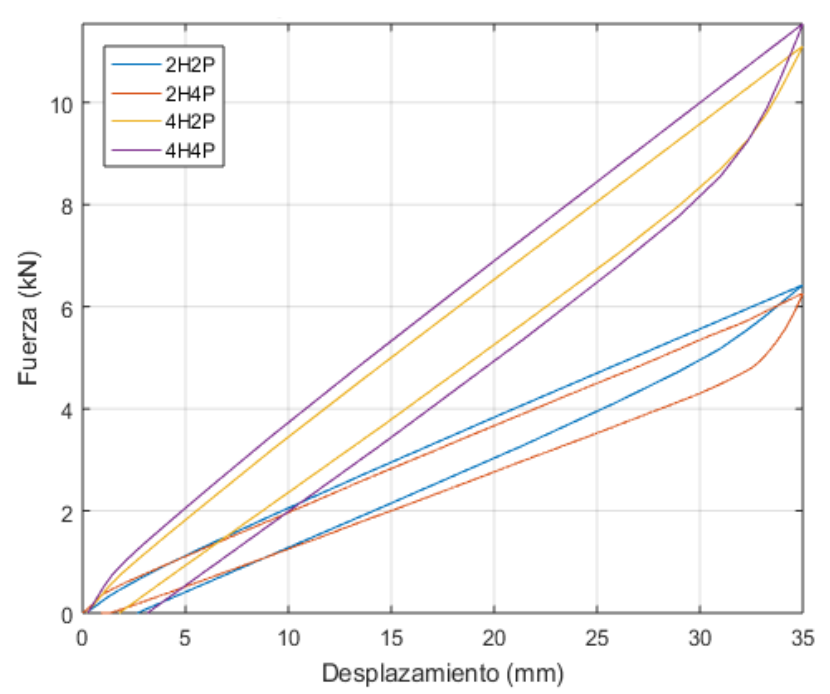

Figura 6. Resultados numéricos de histéresis para muelle.

Los resultados de la energía elástica $E_{o}$, la disipación de energía $E_{r}$, así como de la capacidad de amortiguamiento $\psi$ se presentan en la Tabla 4 . Se puede observar un aumento notable en la disipación de energía mientras que la energía elástica se incrementa ligeramente. Con dos hojas, de la configuración $2 \mathrm{H} 2 \mathrm{P}$ a la $2 \mathrm{H} 4 \mathrm{P}$ la disipación de energía $E_{r}$ aumenta $12 \%$ y la energía elástica $E_{o}$ un $2.1 \%$.

ISSN: 2594-1925 
Cuando se cambia de dos a cuatro hojas, es decir de la configuración $2 \mathrm{H} 4 \mathrm{P}$ a la $4 \mathrm{H} 2 \mathrm{P}$, la disipación de energía $E_{r}$ se incrementa $53 \%$, mientras que la energía elástica $E_{o}$ aumenta $61 \%$. En el rango de cuatro hojas, al pasar de la configuración $4 \mathrm{H} 2 \mathrm{P}$ a la 4H4P, la disipación de energía $E_{r}$ aumenta $55 \%$ mientras que la energía elástica $E_{o}$ aumenta $3 \%$. El resultado de los estudios numéricos de la disipación de energía y de la energía elástica permite advertir que existe un incremento de ambas energías, existiendo una correspondencia con los valores experimentales, aún con sus variaciones.

Tabla 4. Resultados numéricos de la estimación de energía.

\begin{tabular}{cccccc}
\hline Configuración & $\boldsymbol{E}_{\boldsymbol{o}}(\mathrm{J})$ & $\boldsymbol{E}_{\boldsymbol{r}}(\mathrm{J})$ & $\boldsymbol{\psi}$ & $\begin{array}{c}\Delta \boldsymbol{E}_{\boldsymbol{o}} \\
\boldsymbol{\%}\end{array}$ & $\begin{array}{c}\Delta \boldsymbol{E}_{\boldsymbol{r}} \\
\boldsymbol{\%}\end{array}$ \\
\hline 2H2P & 108.049 & 21.775 & 0.20 & - & - \\
2H4P & 110.313 & 24.416 & 0.22 & 2.1 & 12 \\
4H2P & 177.135 & 37.234 & 0.21 & 61 & 53 \\
4H4P & 182.301 & 57.871 & 0.32 & 3 & 55 \\
\hline
\end{tabular}

Comparando los valores de energía elástica para los casos experimental y numérico, se observa que la energía elástica es un poco mayor en el estudio experimental. Esto puede deberse a la geometría no uniforme que presenta el muelle físico, el cual se simula de manera idealizada en el modelo numérico. Los valores obtenidos en disipación de energía de ambos estudios se aproximan entre sí, siendo mayores aquéllos de los ensayos experimentales, como se observa en la Figura 7. Estas diferencias se pueden explicar a partir del montaje experimental, el cual presenta algunas cantidades adicionales de disipación de energía, debido a circunstancias que no se toman en cuenta para el modelo numérico, tales como la fricción entre el muelle y el plato de compresión, la fricción entre los pernos y el canal del muelle, el contacto entre el banco y la máquina universal, entre otros. Esto causa que el valor de la disipación obtenida experimentalmente sea mayor al valor obtenido numéricamente, donde se realizan algunas idealizaciones en torno al montaje del muelle en el chasis del vehículo.

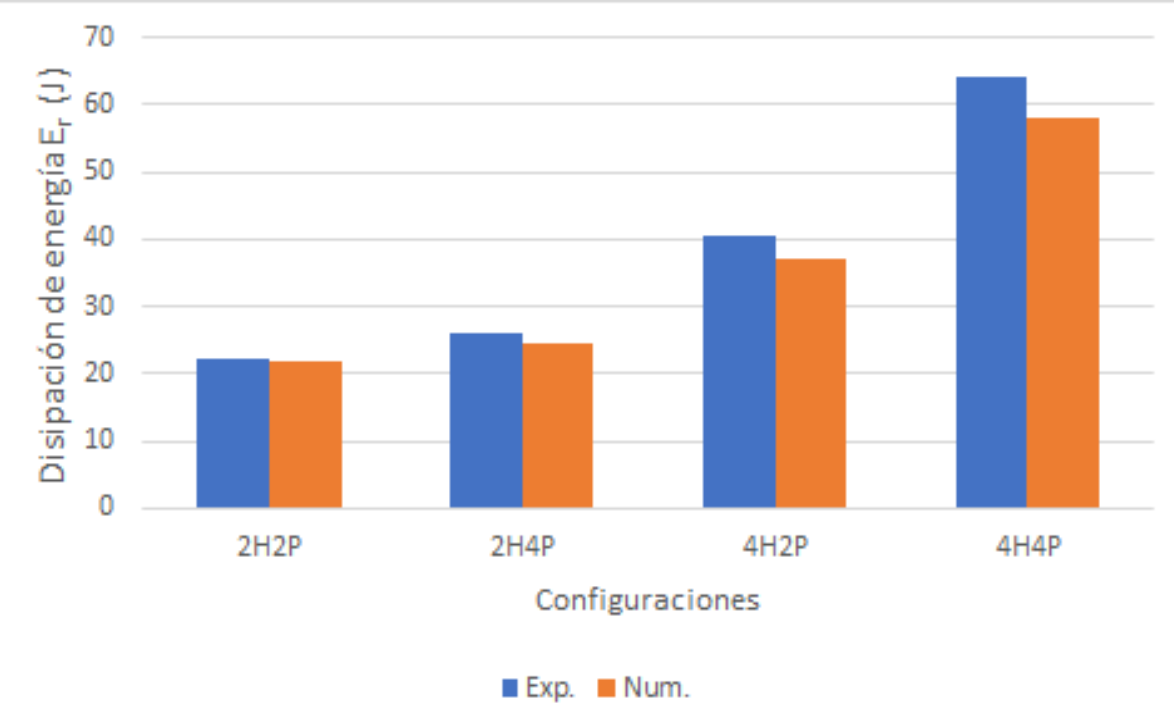

Figura 7. Comparativo entre disipación de energía experimental y numérica. 
En general se observa una buena relación entre los valores numéricos obtenidos por el software y los datos experimentales, apreciándose un patrón de diferencias en la disipación de energía. Se obtuvo un factor de ajuste entre los resultados experimentales y numéricos para cada una de las configuraciones, de acuerdo con la ecuación (4), donde $x$ es la cantidad de hojas que conforma el muelle y $y$ la cantidad de precargas. Este crece en línea recta, como se observa en la Figura 8. Con este factor, los resultados numéricos se ajustan proporcionalmente, considerando las circunstancias experimentales que fueron idealizadas en el modelo numérico. En ese aspecto, se toman en cuenta las últimas tres filas de la Tabla 2 para extender el estudio a otras configuraciones a través del estudio numérico (ver Tabla 5).

Tabla 5. Resultados de disipación de energía del estudio extendido.

\begin{tabular}{cc}
\hline Configuración & $\boldsymbol{E}_{\boldsymbol{r}}(\mathrm{J})$ \\
\hline 3H0P & 20.509 \\
\hline 3H2P & 26.850 \\
\hline 3H4P & 37.411 \\
\hline
\end{tabular}

\section{Factor de ajuste}

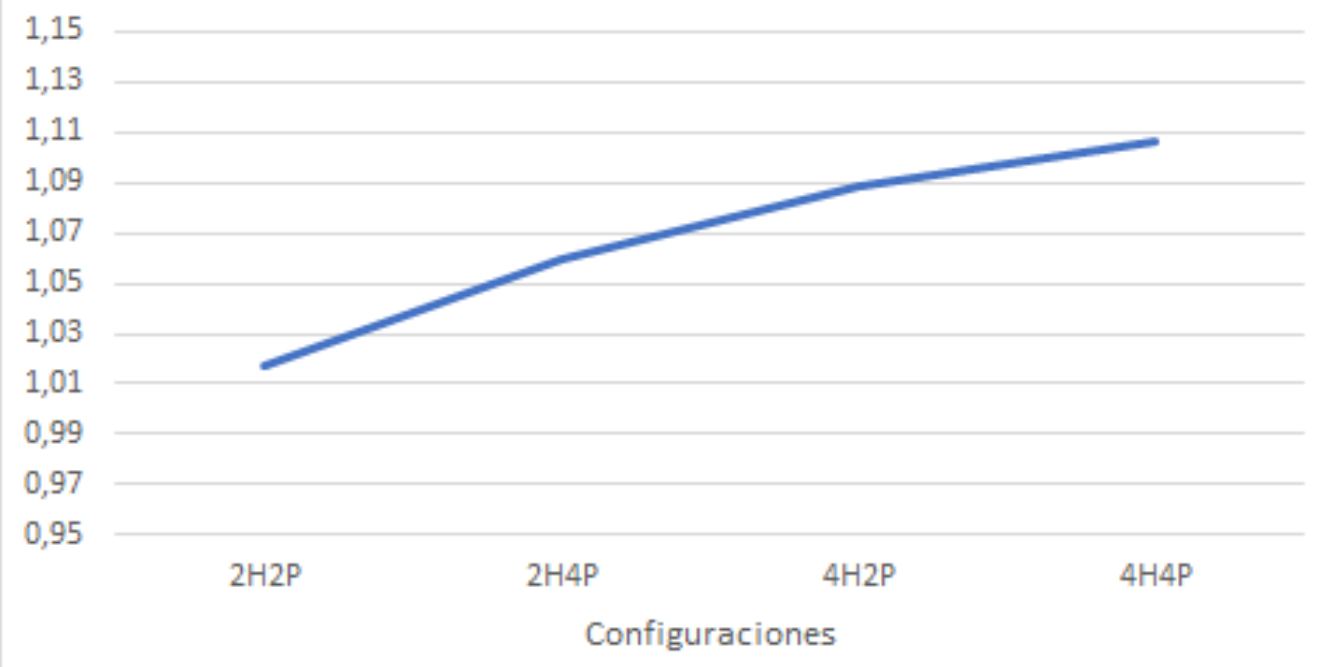

Figura 8. Factores de ajuste entre los resultados experimentales y numéricos para cada una de las configuraciones.

$$
\text { Ajuste }=1.07854+0.011528 x-0.0329904 y+0.00602028 x y
$$

Las gráficas de la Figura 9 muestran la influencia de las variables en la disipación de energía. La curva roja representa la relación obtenida con los resultados numéricos, y la curva azul son los resultados aplicando el factor de ajuste determinado previamente. Se observa que cuando se tiene un número reducido de hojas, el efecto

ISSN: $2594-1925$ 


\section{Revista de Ciencias Tecnológicas (RECIT). Volumen 3 (4): 181-195}

de las precargas en la disipación de energía del muelle es reducido. Sin embargo, cuando se aumenta el número de hojas, el número de precargas tiene una influencia aún mayor en la disipación de energía. Esto es debido a que con mayor número de hojas se tiene mayor número de interacciones de contacto, por lo que hay más superficies deslizándose una sobre otra, lo que origina más fricción y por lo tanto mayor disipación de energía. La ecuación (5) es una regresión obtenida con los datos de las simulaciones numéricas y que corresponde a la curva roja de la Figura 9, donde $E_{r}$ es la disipación de energía en Joules, $x$ la cantidad de hojas que conforma el muelle y $y$ la cantidad de precargas involucradas. Para obtener a curva azul se multiplican las ecuaciones (4) y (5) y se grafica la función resultante.

$$
E_{r}=60.7224-35.8952 x-7.3325 y+6.95083 x^{2}+2.3755 x y+1.32483 y^{2}
$$

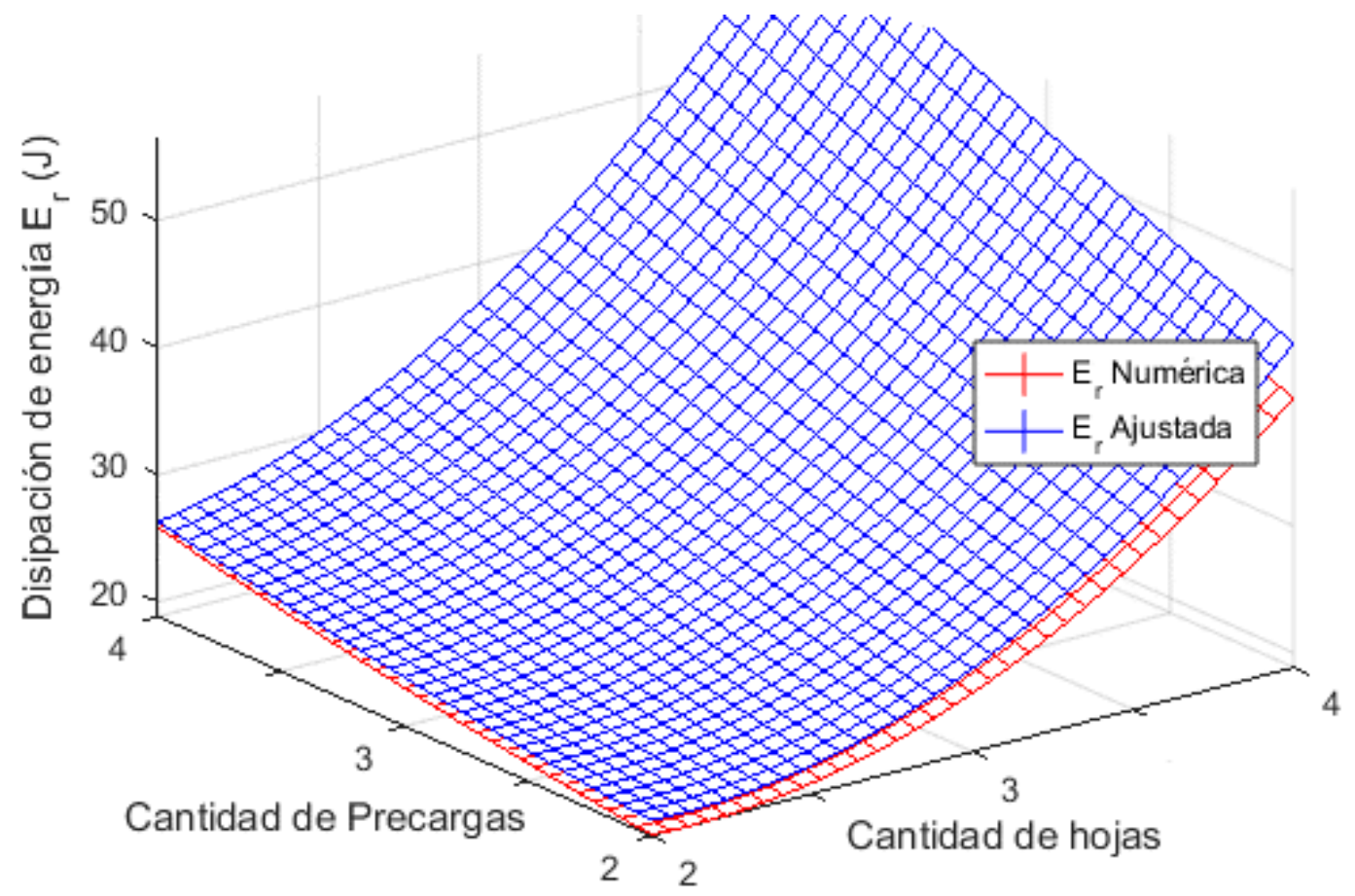

Figura 9. Gráfico de interacción entre hojas y precargas en la disipación de energía.

En la Figura 10 se muestran los valores del área de contacto total y la disipación de energía en un ciclo de carga de los modelos del muelle probados. Como se ha reportado en la literatura [21], las hojas del muelle se deslizan una sobre otra, tanto en el momento de la compresión como en la relajación de la carga, pero que en sentido contrario. Al incrementarse el número de hojas, las áreas en contacto aumentan, y por lo tanto también las zonas en deslizamiento al momento de deformar el muelle, por lo que la disipación de energía sigue una tendencia a incrementarse durante el ciclo de carga semejante al del área de contacto. Se infiere que el incremento en mayor medida del área

ISSN: 2594-1925 
de contacto se da conforme a un mayor número de hojas, mientras que las precargas implementadas incrementan también, aunque en menor medida, esta área de contacto. Esto es debido a que las precargas ejercen una fuerza normal solamente sobre regiones localizadas en el muelle. Los elementos para precarga fueron colocados cerca de los extremos de las hojas del muelle donde, de acuerdo con la literatura [16], se registran los mayores puntos de fricción, es por esto por lo que si se aplican precargas entre más hojas tenga el muelle, mayor es el incremento de la disipación de energía. La implementación de precargas tiene un efecto menor cuando el muelle está formado por pocas hojas, observándose en estos casos muy poco incremento, tanto de la disipación de energía como del área de contacto.

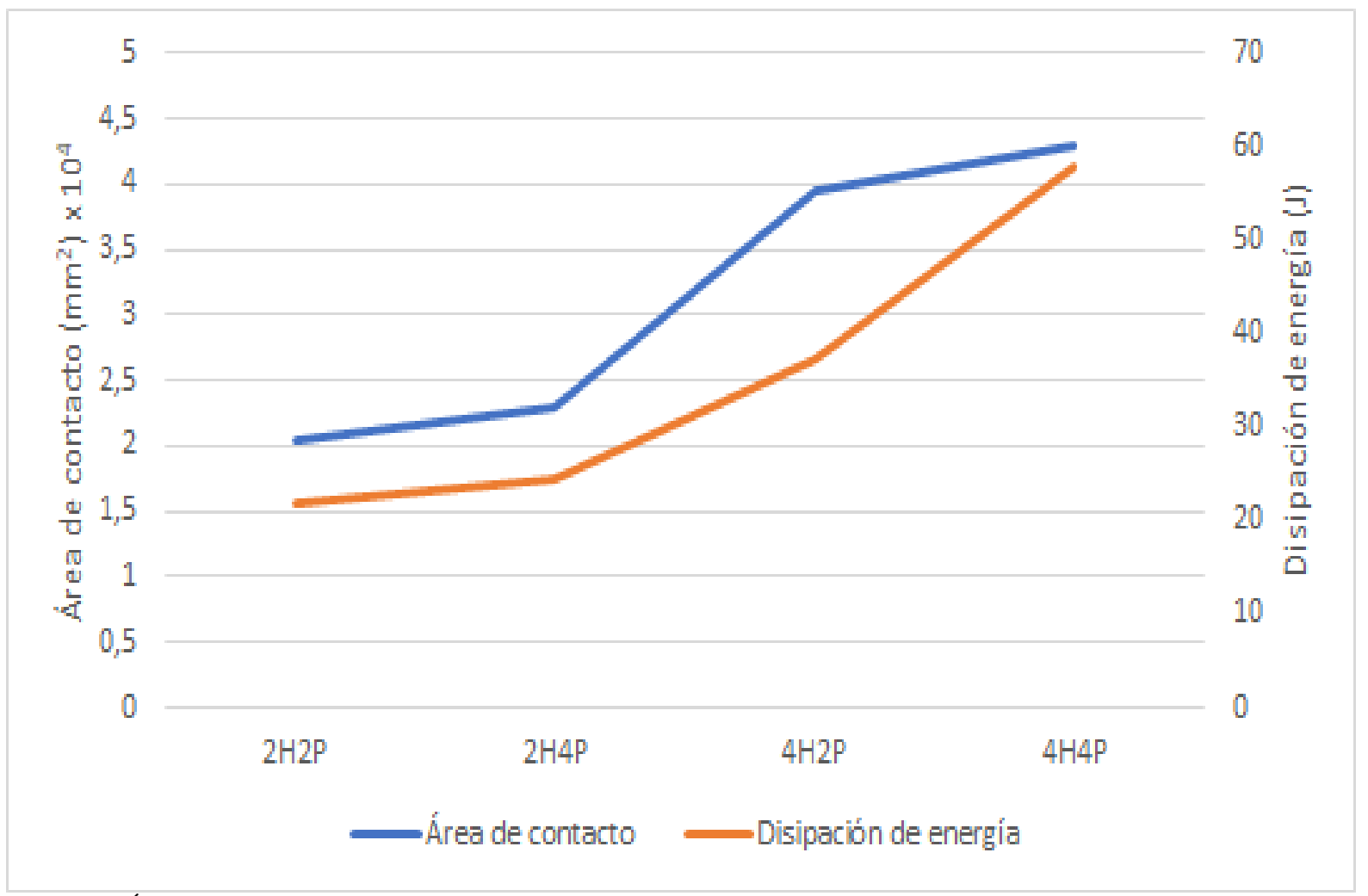

Figura 10. Área de contacto total para las diferentes configuraciones del muelle en un ciclo de carga.

\section{Conclusiones}

Se realizó una evaluación experimental y numérica del efecto de la variación de la precarga de ensamble y de la cantidad de hojas sobre la disipación de energía por fricción de un muelle. Los resultados muestran que un mayor número de hojas incrementa la disipación de energía y la rigidez del muelle. Se observó que la implementación de precargas aumentó la disipación de energía, pero su efecto en el aumento de la rigidez es menor. Por lo tanto, de acuerdo con la aplicación requerida, es posible cambiar la configuración del muelle. En caminos suaves, cuando se conduce con cargas pequeñas, se busca un amortiguamiento mínimo y por lo tanto una

ISSN: 2594-1925 
menor fricción, para un mejor confort de los pasajeros. En cambio, una mejor estabilidad del vehículo se obtiene cuando se incrementa la fricción, con un amortiguamiento mayor para cargas grandes. Esto puede dar pauta para el desarrollo de técnicas como la de Shinbori y Matsuoka [22], quienes inventaron un sistema semiactivo que controla el apriete de las hojas en uno de los extremos del muelle. Dependiendo de las necesidades del vehículo, ya sea confort o estabilidad, es capaz de disminuir o aumentar la fricción, respectivamente.

Se obtuvo un modelo empírico que relaciona la capacidad de disipación de energía del muelle con la cantidad de hojas y de precargas. Este puede usarse para asistir el proceso de diseño de suspensiones de camiones de carga, buscándose implementar condiciones de apriete con algún sistema semiactivo. Se le aplicó un factor de ajuste con el fin de incluir los efectos de las simplificaciones realizadas.
Se sugiere estudiar las configuraciones probadas bajo condiciones de fatiga y evaluar la influencia de las precargas en las frecuencias naturales del muelle. Se recomienda evaluar otros tipos de precargas, mediante las cuales se aborde otra forma de contacto en el apriete, o con alguna precarga axial al muelle. También se aconseja evaluar la disipación de la energía mediante un diseño experimental del tipo $3^{\mathrm{k}}$ o mixto, ya que estos permiten un mayor número de niveles de tratamiento de los factores que el diseño factorial $2^{2}$.

\section{Agradecimientos}

A los integrantes del Laboratorio de Vibraciones y Esfuerzos del Departamento de Ingeniería Mecánica del TecNM/CENIDET por el apoyo en las pruebas y en comentarios e ideas para este trabajo. Al CONACYT por la beca número 701125. Al Dr. Arturo Abúndez Pliego por sus sugerencias para la redacción y conclusión de este artículo. 
Revista de Ciencias Tecnológicas (RECIT). Volumen 3 (4): 181-195

\section{Referencias}

[1] A. G. Piersol y T. L. Paez, Harris' shock and vibration handbook. McGraw-Hill, 2010.

https://www.accessengineeringlibrary.com/con tent/book/9780071508193

[2] J. C. Dixon, The Shock Absorber Handbook. Chichester, UK: John Wiley \& Sons, Ltd, 2007. https://www.wiley.com/enus/The+Shock+Absorber+Handbook\%2C+2nd +Edition+-p-9780470510209

[3] J. R. Matienzo y L. O. Pereiro, "Modelo de un muelle de ballestas considerando la fricción entre hojas", Ing. Mecánica, vol. 9, núm. 1, pp. 15-28, 2006. https://www.semanticscholar.org/paper/Model o-de-un-muelle-de-ballestas-considerando-la$\% 2 \mathrm{~F} \% 2 \mathrm{~F}-\mathrm{Matienzo}-$

Pereiro/a9f1370b88afee15121f06de72f6f6e94d 3e2f48

[4] Young-Jin Yum, "Frictional behavior of automotive leaf spring", en Proceedings KORUS 2000. The 4th Korea-Russia International Symposium On Science and Technology, 2000, vol. 3, pp. 5-10. https://www.doi.org/10.1109/KORUS.2000.86 6051.

[5] A. González Rodríguez, J. M. Chacón, A. Donoso, y A. G. González Rodríguez, "Design of an adjustable-stiffness spring: Mathematical modeling and simulation, fabrication and experimental validation", Mech. Mach. Theory, vol. 46, núm. 12, pp. 1970-1979, dic. 2011. https://www.doi.org/10.1016/j.mechmachtheor y.2011.07.002.

[6] S. Karditsas, G. Savaidis, y M. Malikoutsakis, "Advanced leaf spring design and analysis with respect to vehicle kinematics and durability", Int. J. Struct. Integr., vol. 6, núm. 2, pp. 243-258, abr. 2015. https://www.doi.org/10.1108/IJSI-11-20130044.

[7] M. Malikoutsakis, G. Savaidis, A. Savaidis, C. Ertelt, y F. Schwaiger, "Design, analysis and multi-disciplinary optimization of high-performance front leaf springs", Theor. Appl. Fract. Mech., vol. 83, pp. 42-50, jun.
2016.

https://www.doi.org/10.1016/j.tafmec.2016.01. 008.

[8] Z. Xu, L. Hong, X. L. Wang, y C. S. Ding, "Study on the Influence of the Shape of Leaf Spring on the Stress and the Kinematic Characteristics", J. Eng. Res. Appl., vol. 8, núm. 6, pp. 13-21, 2018. https://www.doi.org/10.9790/96220806031321.

[9] M. M. Shokrieh y D. Rezaei, "Analysis and optimization of a composite leaf spring", Compos. Struct., vol. 60, núm. 3, pp. 317-325, may

2003.

https://www.doi.org/10.1016/S02638223(02)00349-5.

[10] K. Kumar y A. M L, "Simulation For Optimized Modelling of En45A Leaf Spring", Int. J. Recent Adv. Mech. Eng., vol. 4, núm. 3, pp. 129-142, ago. 2015. https://www.doi.org/10.14810/ijmech.2015.431 0 .

[11] D. Ashok Kumar y A. Kalam SD, "Design, Analysis and Comparison between the Conventional Materials with Composite Material of the Leaf Springs", Fluid Mech. Open Access, vol. 03, núm. 01, 2016. https://www.doi.org/10.4172/2476-

2296.1000127.

[12] K. Ashwini y C. V. Mohan Rao, "Design and Analysis of Leaf Spring using Various Composites - An Overview", Mater. Today Proc., vol. 5, núm. 2, pp. 5716-5721, 2018.

https://www.doi.org/10.1016/j.matpr.2017.12.1 66.

[13] P. Qin, G. Dentel, y M. Mesh, "Multileaf spring and Hotchkiss suspension $\mathrm{CAE}$ simulation", en ABAQUS Users' Conference, 2002, pp. 1-14. http://www.simulia.com/download/solutions/au tomotive_cust\%20references/chassis_mulitleaf _auc02_chrysler.pdf

[14] B. Kadziela, M. Manka, T. Uhl, y A. Toso, "Validation and optimization of the leaf spring multibody numerical model", Arch. Appl. Mech., vol. 85, núm. 12, pp. 1899-1914, dic. 2015. https://www.doi.org/10.1007/s00419015-1024-5. 
Revista de Ciencias Tecnológicas (RECIT). Volumen 3 (4): 181-195

[15] W. Krason y J. Wysocki, "Numerical studies of suspension system with double spring loaded using the force pulse", J. KONES, vol. 25, núm. 1, pp. 241-248, 2018. https://www.doi.org/10.5604/01.3001.0012.24 $\underline{73}$.

[16] W. Krason y J. Wysocki, "Investigation of friction in dual leaf spring", J. Frict. Wear, vol. 38, núm. 3, pp. 214-220, may 2017. https://www.doi.org/10.3103/S1068366617030 096.

[17] W. Krason, J. Wysocki, y Z. Hryciow, "Dynamics stand tests and numerical research of multi-leaf springs with regard to clearances and friction", Adv. Mech. Eng., vol. 11, núm. 5, pp. 1-13, may 2019. https://www.doi.org/10.1177/16878140198533 53.

[18] S. A. E. HS788, "Manual on Design and Application of Leaf Springs", Soc. Automot. Eng., https://www.sae.org/publications/books/conten t/hs-788/

[19] J. K. Budynas, Richard G.; Nisbett, Diseño en ingeniería mecánica de Shigley, 8a ed. México, D.F.: McGraw-Hill, 2012. http://www1.frm.utn.edu.ar/electromecanica/m aterias\%20pagina\%20nuevas/elementoMaquin a/material/libroCabecera.pdf

[20] Imperial Supplies LLC, "Fastener Torque Charts". https://www.imperialsupplies.com/static/charts [21] E. Zahavi, "Analysis of a contact problem in leaf springs", Mech. Res. Commun., vol. 19, núm. 1, pp. 21-27, ene. 1992, https://www.doi.org/10.1016/00936413(92)90006-V.

[22] T. Shinbori y S. Matsuoka, "Apparatus for controlling friction between leaf springs of a laminated leaf spring assembly", 4,456,232, 1984. https://patents.justia.com/patent/4463936

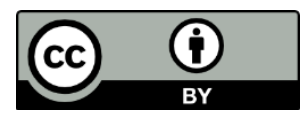

Este texto está protegido por una licencia $\underline{\text { Creative Commons 4.0 }}$

Usted es libre para Compartir — copiar y redistribuir el material en cualquier medio o formato — y Adaptar el documento — remezclar, transformar y crear a partir del material - para cualquier propósito, incluso para fines comerciales, siempre que cumpla la condición de:

Atribución: Usted debe dar crédito a la obra original de manera adecuada, proporcionar un enlace a la licencia, e indicar si se han realizado cambios. Puede hacerlo en cualquier forma razonable, pero no de forma tal que sugiera que tiene el apoyo del licenciante o lo recibe por el uso que hace de la obra. 